\title{
Saponosides stéroïdiques de l'aubergine (Solanum melongena L.) II. Variations des teneurs liées aux conditions de récolte, aux génotypes et à la quantité de graines des fruits
}

\author{
S. Aubert ${ }^{1}$, M. C. Daunay ${ }^{2}$ et E. Pochard 2 \\ 1 INRA, centre de recherches d'Avignon, station de technologie des produits végétaux, domaine Saint-Paul, BP 9184140 \\ Montfavet; \\ 2 INRA, centre de recherches d'Avignon, station d'amélioration des plantes maraíchères, domaine Saint-Paul, BP 9184140 \\ Montfavet, France
}

(reçu le 28 juillet 1987, accepté le 24 avril 1989)

Résumé - Les glycoalcaloïdes et saponosides stéroïdiques non azotés du type furostanol constituent des critères de la qualité gustative et sanitaire des fruits de l'aubergine. Les teneurs en ces substances sont d'autant plus élevées que les récoltes sont espacées et, par conséquent, que le stade de maturité physiologique des fruits est avancé. Les fruits récoltés en fin de culture présentent généralement de fortes teneurs. Les variations quantitatives des saponosides sont accompagnées de différences qualitatives liées à la nature des substituants hétérosidiques des aglycones.

L'influence du génotype sur la teneur en saponosides des fruits n'est établie que pour les variétés d'aubergine ou d'autres espèces de Solanum voisines dont la saveur typique est particulièrement forte ou douce. Dans les fruits de saveurs «jntermédiaires", l'appréciation de la part "génétique» est généralement difficile à dissocier des facteurs culturaux et des conditions de récolte, surtout dans les produits prélevés au stade commercial dont les teneurs en saponosides sont relativement faibles.

La présence de graines n'est généralement pas favorable à l'agrément gustatif. Quelques essais sur des variétés à tendance parthénocarpique, fécondées de différentes façons, n'ont pas permis d'établir précisément l'influence sur le goût de la quantité de graines. Certains fruits sans graines ont une saveur amère excessive. Mais ces résultats ont été obtenus dans des conditions de cultures particulièrement défavorables : fins de récolte de productions sous serre plastique, hautes températures et hygrométrie insuffisante. D'autres facteurs peuvent aussi favoriser l'accumulation des saponosides dans les fruits.

solanacée - saponine - récolte - parthénocarpie - serre

Summary - Steroidic saponins from egg-plant (Solanum melongena L.) II - Effects of cultural conditions, genotypes and parthenocarpy. Glycoalkaloids and steroidic saponins without nitrogen, of the furostanol type, are determinants of the sapid and sanitary qualities of egg-plant fruit.

Saponin contents were higher with lengthened harvesting periods which lead to advanced physiological maturity of the fruits. Fruit harvested at the end of season generally have high saponin contents. Quantitative saponin variations were associated with qualitative differences linked to the heterosidic composition of glycoalkaloids.

Genotype factors affecting saponin contents are only established for genuine egg-plant varieties or solanaceous species close to Solanum melongena $L$. showing significant differences in composition between strong and sweet sapidity. However, for fruit with "intermediate" sapidity, the characteristics attributed to genotype were often difficult to dissociate from cultivation factors and production conditions, especially in products tested at the marketing stage when saponin contents are relatively low.

Seed content is detrimental to sensory quality. The few trials performed with different pollination conditions, on varieties with parthenocarpic tendency, did not confirm this observation. Thus, some seedless fruits exhibited an excessively bitter taste. But these results were obtained with particularly unfavourable cultivation conditions : fruits harvested at the end of season under plastic green-houses, high temperature and low hydric conditions.

Other factors also favour the accumulation of saponins in fruit.

In practice, precocity and harvest frequency gave fruits of better quality. 


\section{INTRODUCTION}

Dans la première partie de cette étude (Aubert et al., 1989), nous avons réalisé une synthèse bibliographique concernant les saponosides stéroïdiques (glycoaicaloïdes et saponosides non azotés) du fruit de l'aubergine : structures moléculaires, méthodes d'extraction et d'analyse, propriétés gustatives et sanitaires. L'influence déterminante de fortes teneurs des fruits en saponosides sur leur amertume a été confirmée expérimentalement. Des limites d'acceptabilité gustative ont été données sur des variétés typiques et leurs croisements. Des teneurs élevées ont été trouvées chez les fruits "vieux", ayant dépassé le stade commercial de récolte précoce, et dans la partie de la pulpe renfermant les graines.

Par ailleurs, il a été établi que la couleur blanche des fruits n'est pas liée systématiquement à leur faible teneur en saponosides.

Toutefois, l'enrichissement des fruits en saponosides peut être influencé par des facteurs culturaux et agroclimatiques (Khristov, 1975). C'est une observation de pratique courante que les fins de production en serre, comme au champ, donnent parfois des fruits inacceptables sur le plan gustatif. Juvik et al. (1982), Man et al. (1980), Yaniv et al. (1984) ont montré que les saponosides interviennent dans la réponse des plantes aux variations climatiques. De plus, Defago (1977) et Rowan et al. (1983) ont étudié leur implication dans les mécanismes de résistance aux attaques parasitaires, notamment fongiques.

Dans cette seconde partie, nous poursuivrons l'étude des facteurs responsables de la variation des teneurs en saponosides stéroïdiques, sur des lots de fruits de diverses variétés et cultures. Tout d'abord, nous rapporterons l'évolution des teneurs en divers saponosides au cours des cueillettes successives de 5 variétés connues, sur une même plantation et pour une culture estivale de plein champ. Ensuite, nous étudierons la variabilité des teneurs due aux facteurs génétiques, en comparant 12 variétés commerciales, ou de création récente, sur plusieurs années de récolte. Trois autres espèces de Solanum, phylogénétiquement proches de l'aubergine, ont été comparées aux aubergines traditionnelles pour situer leurs teneurs par rapport aux niveaux admis sur les plans gustatif et sanitaire. Enfin, nous utiliserons les effets de la parthénocarpie naturelle de quelques variétés pour contrôler l'influence de la présence plus ou moins abondante de graines sur l'amertume des fruits.

\section{MATÉRIEL ET MÉTHODES}

\section{Matériel végétal}

Les fruits proviennent de cultures expérimentales mises en place par la station d'amélioration des plantes maraîchères de I'INRA de Montfavet. Les génotypes étudiés peuvent être classés en trois groupes.

\section{Les variétés commerciales et lignées de I'INRA}

A ce groupe appartiennent des variétés cataloguées en France (Joubert \& Compagnon, 1974) : "Violette de Barbentane", "Ronde de Valence", "Dourga", "F1 Baluroi", "F1 Bonica", "F1 Giniac» et des variétés étrangères : soit hollandaises ( $F 1$ Dobrix»), soit extrême-orientales ("Liu Yé Qié" et "Shinkuro"). Les lignées issues de l'INRA sont : "Athéna" et "Jordanie».

\section{Les génotypes à tendance parthénocarpique}

"Claresse" (variété hollandaise), "Konzervnij" (origine soviétique) et «Trachietz» (origine bulgare) forment spontanément des fruits sans graines ou avec très peu de graines, en culture de printemps sous abri froid.

\section{Les espèces de Solanum, voisines de $S$. melongena $\mathrm{L}$.}

Nous avons utilisé une espèce spontanée, $S$. sodomeum auct. non L. (ou $S$. hermanii, d'après Hepper, 1979), et 2 espèces cultivées pour leurs fruits, consommés en particulier en Afrique : $S$. macrocarpon $L$. et $S$. aethiopicum L. Selon la nomenclature mise au point par Lester \& Niakan (1986), les cultivars de $S$. aethiopicum que nous avons utilisés appartiennent, l'un au groupe "gilo" (anciennement appelé $S$. anomalum), le second au groupe "aculeatum" (anciennement appelé $S$. integrifolium); le dernier cultivar est le «Djackattou» mauritanien, qui appartient au groupe “Kumba" (De Bon, 1984). Pour celui-ci, nous avons utilisé un lot de fruits séchés fourni par l'Institut de recherche en agronomie tropicale (CIRAD-GERDAT) et le Centre national de développement agricole de Mauritanie.

\section{Echantillonnage et comparaisons variétales}

Les fruits sont généralement cueillis au stade commercialisable (c'est-à-dire à la fin de la période d'élongation rapide); parfois ce stade est légèrement dépassé. Les analyses ont été effectuées en fonction des disponibilités présentes en fruits sur diverses parcelles expérimentales.

L'essai mis en place en plein champ, afin d'étudier l'évolution de la teneur en saponosides au cours de l'été, porte sur les 5 variétés : “Ronde de Valence", "Dourga", "Athéna", "Trachietz" et «F1 Baluroi". Dix récoltes ont été effectuées entre le 7 juillet et le 30 septembre 1984. Compte tenu du nombre limité de 
plantes (4 répétitions de 5 plantes par variété), l'échantillonnage a été restreint à 5 - 8 fruits (cueillis au stade commercialisable) par variété et par récolte.

L'essai d'analyse de l'aptitude à la parthénocarpie de 3 variétés : "Claresse», “Konzervnij» et "Trachietz" a été mis en place en tunnel froid au printemps. Ces variétés sont capables de former des fruits issus de fleurs sans ou à faible pollinisation; alors que les variétés courantes nécessitent une bonne pollinisation pour fructifier. Cet essai a permis de comparer la teneur en saponosides de 3 types de fruits :

- fruits parthénocarpiques (sans graines), issus de fleurs castrées;

- fruits contenant peu de graines, issus de fleurs laissées en pollinisation naturelle (en tunnel froid, celle-ci est très mauvaise);

- fruits contenant beaucoup plus de graines, issus de fleurs "vibrées" (ce traitement améliore la pollinisation).

\section{Méthodes d'analyse des saponosides}

Les glycoalcaloïdes sont dosés par 2 techniques distinctes, la colorimétrie et la chromatographie sur couche mince (CCM); cette deuxième technique permet de séparer les différents hétérosides de la solasodine. Elle est aussi utilisée pour évaluer les saponosides non azotés du type furostanol (Aubert et al., 1989).

\section{Autres critères de qualité}

L'éclaircissement de la coloration pourpre de l'épiderme permet de repérer l'hétérogénéité des stades de maturité physiologique des fruits d'un même échantillonnage (Aubert, 1971). Sur ce critère, la pigmentation anthocyanique est exprimée en unités de densité optique (UDO) à $550 \mathrm{~nm}$ par $\mathrm{cm}^{2}$ de surface d'épiderme prélevé.

La quantité de graines présentes dans la pulpe est estimée visuellement; leur coloration et leur dureté renseignent aussi sur le stade de maturité du fruit.

\section{RÉSULTATS}

\section{Variations de composition au cours des récoltes}

La teneur des fruits en glycoalcaloïdes (Fig. 1) et en saponosides non azotés du type furostanol (Fig. 2) est très variable d'une récolte à l'autre. Plus particulièrement, les teneurs en glycoalcaloïdes augmentent notablement en fin de saison (Fig. 1). Cet accroissement n'est pas perçu sur les saponosides non azotés du type furostanol (Fig. 2).

La séparation par chromatographie sur couche mince (Fig. 3) des hétérosides de la solasodine met en évidence pour la lignée "Athéna", prise en exemple, l'existence de changements impor- tants de composition en glycoalcaloïdes au cours du temps. Le nombre de taches révélées par CCM augmente entre les premières et les dernières récoltes. On note une différence qualitative (position des taches) et quantitative (surface et intensité) entre les taches correspondant à des extraits de fruits prélevés visiblement plus "vieux» (récolte 5) ou plus «jeunes" (récolte 6), indépendamment de la date de cueillette. II semble donc que le stade physiologique de maturité à la récolte joue un rôle majeur dans le métabolisme des glycoalcaloïdes.

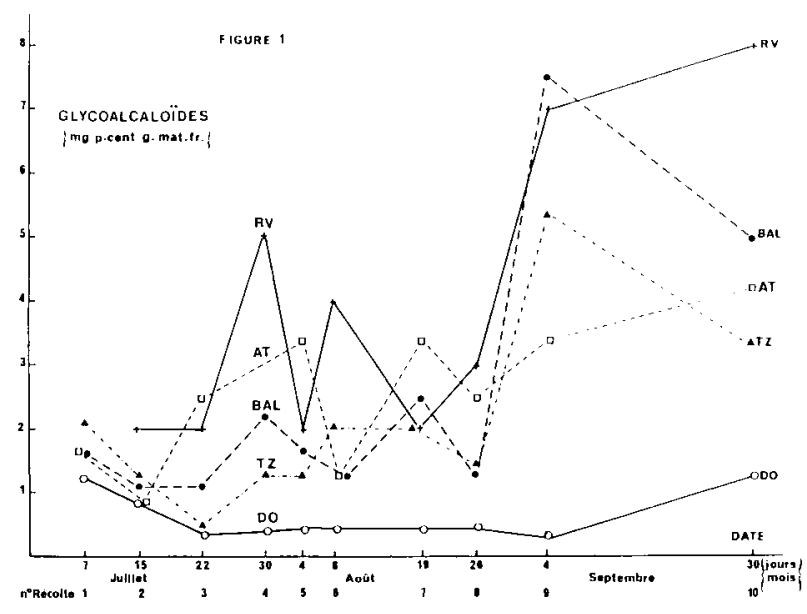

Fig. 1. Evolution des teneurs en glycoalcaloïdes au cours des récoltes successives de diverses variétés d'aubergines. "Ronde de Valence" = RV+—; "Dourga" = DO $\mathrm{O}-\mathrm{O}$; "F1 Baluroi" = BAL $---\rightarrow$;Athena" = AT 口---- - ; Trachietz» = TZ $\Delta-\cdot \cdot \cdot \cdot \cdot-\mathbf{A}$.

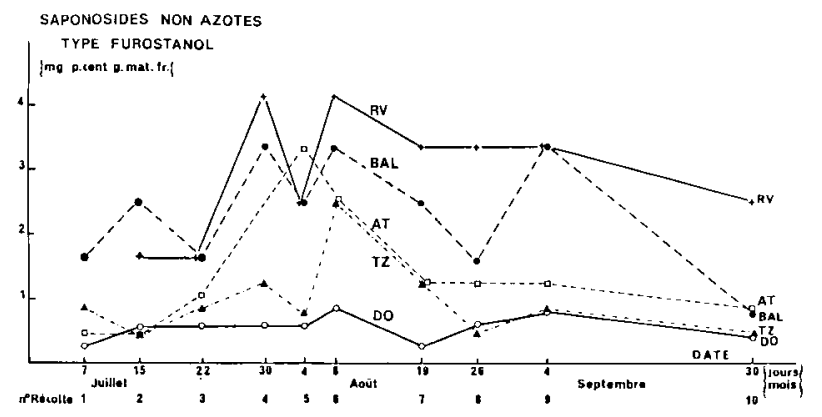

Fig. 2. Evolution des teneurs en saponosides non azotés (SNA) du type furostanol au cours des cueillettes successives de diverses variétés d'aubergines.

"Ronde de Valence" = RV+ - +; "Dourga" = DO O - ; 1 Baluroi" = BAL - - "Athena" = AT $\square-\ldots--\square ;$ Trachietz" = TZ A- . - . . - $\mathbf{\Delta}$.

\section{Variabilité génétique}

On retrouve sur l'ensemble des récoltes la confirmation de nos premiers résultats (Aubert et al., 1989) concernant les variétés les plus contrastées. "Ronde de Valence", de sapidité forte, présente généralement les plus fortes teneurs en saponosides (Figs 1 et 2), tandis que "Dourga", 
de saveur douce, produit les plus faibles. Un classement des autres variétés, à valeurs intermédiaires s'avère difficile. En effet, quelle que soit la technique de dosage utilisée, colorimétrie ou CCM, (Tableau I), les écarts types sur les moyennes variétales sont importants. Néanmoins, à partir des 2 références typiques précédentes, on peut distinguer 3 groupes variétaux : «Ronde de Valence», «Jordanie», «F1 Giniac» et "Violette de Barbentane" sont les variétés les plus riches, tandis que «Dourga» et «Shinkuro» ont les plus faibles concentrations; les autres variétés : «F1 Baluroi», «F1 Bonica», «Trachietz", "Liu Ye Qié», "F1 Dobrix» et "Athéna» ont des teneurs intermédiaires.

D'autre part, les variétés diffèrent plus entre elles pour les teneurs en glycoalcaloïdes (GA) que pour les teneurs en saponosides non azotés (SNA) du type furostanol (Tableau II).

La variabilité génétique au sein de l'espèce Solanum melongena (aubergine) couvre, pour les 12 variétés considérées (Tableau II), une gamme de teneurs de 1 - $8 \mathrm{mg}$ (pour $100 \mathrm{~g}$ de matière fraîche) en glycoalcaloïdes et un peu moins (1 - 3,5 mg) en saponosides non azotés, type furostanol. Ces valeurs moyennes relativement faibles correspondent à des lots généralement récoltés au stade commercial.

Les autres espèces de Solanum (Tableau II) présentent par contre des teneurs beaucoup plus variables pour les glycoalcaloïdes. S. sodo- meum est particulièrement riche $(225 \mathrm{mg})$ alors que les autres ont des teneurs voisines de celles de l'aubergine. Toutes ces espèces sont beaucoup plus riches que l'aubergine en SNA type furostanol (de l'ordre de 10 fois plus). Par ailleurs, ces saponosides non azotés possèdent une plus grande diversité de radicaux hétérosides, révélée par le plus grand nombre de taches obtenues par CCM (Tableau II). Une analyse organoleptique montre que ces espèces contiennent beaucoup plus de graines que Solanum melongena. Leur saveur amère est typique et très forte.

\section{Influence de la quantité de graines présente dans les fruits}

La richesse des fruits en glycoalcaloïdes et en saponosides non azotés ne dépend pas du nombre de graines qu'ils contiennent (Tableau III), pour les 3 variétés étudiées. Les teneurs des fruits sans graines (issus de fleurs castrées) sont voisines de celles des fruits qui contiennent quelques graines ou beaucoup. Les variations, importantes selon les essais et suivant les techniques de mesure, ne permettent pas de mettre en évidence l'influence de la parthénocarpie, plus ou moins apparente. On notera que les teneurs en glycoalcaloïdes de tous les fruits de cette expérience sont particulièrement élevées (Tableau III, comparé aux Tableaux I et II).

Tableau I. Glycoalcaloïdes de différentes variétés d'aubergine : variabilité selon les lots et la technique de dosage.

\begin{tabular}{|c|c|c|c|c|c|c|c|}
\hline \multirow{2}{*}{$\begin{array}{l}\text { Variétés } \\
\text { lignées }\end{array}$} & \multirow{2}{*}{$\begin{array}{c}\text { Nombre } \\
\text { de lots }\end{array}$} & \multicolumn{3}{|c|}{ Colorimétrie (a) } & \multicolumn{3}{|c|}{$\operatorname{CCM}(a)$} \\
\hline & & Moyenne & Ecart type & $\operatorname{Maxi}(b)$ & Moyenne & Ecart type & Maxi (b) \\
\hline Ronde de Valence & 8 & 6,2 & 3,2 & 11 & 7,2 & 2,7 & 12 \\
\hline Jordanie & 5 & 7,7 & 4,4 & 13 & 8,3 & 2,6 & 12 \\
\hline Giniac $(F 1)$ & 4 & 7,6 & 5,8 & 15 & 6,1 & 4,3 & 10 \\
\hline \multicolumn{8}{|l|}{ Violette de } \\
\hline Barbentane (LF3) & 9 & 6,4 & 8,2 & 25 & 6,7 & 8,7 & 28 \\
\hline Baluroi (F1) & 6 & 5,7 & 6,2 & 18 & 6,4 & 6,8 & 20 \\
\hline Bonica (F1) & 7 & 5,6 & 8,4 & 24 & 5,9 & 6,5 & 15 \\
\hline Tranchietz & 8 & 3,5 & 4,8 & 15 & 4,8 & 5,5 & 18 \\
\hline Liu ye qié & 4 & 2,9 & 0,9 & 4 & 4,1 & 1,2 & 5 \\
\hline Dobrix (F1) & 3 & 2,2 & 1,8 & 4 & 4,6 & 5,5 & 11 \\
\hline Athéna & 4 & 2 & 2,3 & 5 & 3,1 & 3,3 & 8 \\
\hline Dourga & 5 & 1,7 & 1,5 & 4,5 & 1,7 & 1,0 & 3 \\
\hline Shinkuro & 4 & 0,9 & 0,5 & 2 & 0,8 & 0,4 & 1,5 \\
\hline Moyenne/variétés & & 4,4 & 2,4 & 11,7 & 5,0 & 2,3 & 12 \\
\hline
\end{tabular}

Conditions d'analyse et observations : (a) Teneurs en $\mathrm{mg}$ de solanine étalon pour $100 \mathrm{~g}$ de matière fraiche; (b) les teneurs maximales correspondent a des lots de maturité commerciale souvent dépassé; les graines sont dures et les fruits de sapidité excessive. 
Tableau II. Saponosides stéroïdiques des fruits de diverses solanacées cultivées.

\begin{tabular}{|c|c|c|c|c|c|c|c|}
\hline \multirow[t]{2}{*}{$\begin{array}{l}\text { Solanacées } \\
\text { Attribution botanique } \\
\text { Variété (observations) }\end{array}$} & \multirow[t]{2}{*}{ (nb de } & \multicolumn{3}{|c|}{$\begin{array}{c}\text { Glycoalcaloïdes } \\
\text { Teneurs par colorimétrie } \\
\text { (nombre de taches CCM) }\end{array}$} & \multicolumn{3}{|c|}{$\begin{array}{c}\text { Saponosides furostanol } \\
\text { (SNA) par CCM } \\
\text { (nombre de taches) }\end{array}$} \\
\hline & & $\begin{array}{c}\text { Moyenne } \\
\pm E T(a)\end{array}$ & $\begin{array}{l}\text { Maxi } \\
\text { (b) }\end{array}$ & $\begin{array}{l}\text { Nombre } \\
\text { de taches } \\
\text { CCM }\end{array}$ & $\begin{array}{l}\text { Moyenne } \\
\quad \pm E T\end{array}$ & $\begin{array}{l}\text { Maxi } \\
\text { (b) }\end{array}$ & $\begin{array}{l}\text { Nombre } \\
\text { de taches } \\
\text { CCM }\end{array}$ \\
\hline \multicolumn{8}{|l|}{$\begin{array}{l}\text { Solanum melongena L. } \\
\text { Variétés }\end{array}$} \\
\hline Ronde de Valence & (8) & $6,7 \pm 2,7$ & 11,5 & 3 & $2,9 \pm 1,2$ & 4 & 3 \\
\hline Jordanie & (5) & $8,0 \pm 3,2$ & 12 & 4 & $3,4 \pm 0,9$ & 4 & 3 \\
\hline Giniac (F1) & (4) & $6,5 \pm 4,6$ & 12 & 4 & $3,4 \pm 1,9$ & 6 & 2 \\
\hline Violette de Barbentane & (9) & $6,5 \pm 8,4$ & 26,5 & 3 & $2,0 \pm 0,8$ & 3,7 & 2 \\
\hline Baluroi (F1) & (6) & $6,0 \pm 6,4$ & 19 & 4 & $2,3 \pm 1,0$ & 4 & 2 \\
\hline Bonica (F1) & (7) & $5,7 \pm 7,1$ & 19,5 & 4 & $2,5 \pm 2,6$ & 8 & 3 \\
\hline Trachiez & (8) & $4,1 \pm 5,1$ & 16,5 & 4 & $1,6 \pm 0,9$ & 4 & 3 \\
\hline Liu ye quié & (4) & $3,5 \pm 0,3$ & 4 & 4 & $2,1 \pm 0,9$ & 2,6 & 2 \\
\hline Dobrix (F1) & (3) & $3,4 \pm 3,6$ & 7,5 & 3 & $1,4 \pm 0,8$ & 2,2 & 2 \\
\hline Athéna & (4) & $2,6 \pm 2,8$ & 6,5 & 4 & $1,8 \pm 1,8$ & 3,6 & 1 \\
\hline Dourga & (5) & $1,7 \pm 1,2$ & 3,5 & 2 & $0,9 \pm 0,4$ & 1,5 & 2 \\
\hline Shinkuro & (4) & $0,9 \pm 0,5$ & 1,5 & 3 & $2,3 \pm 1,6$ & 4 & 3 \\
\hline \multicolumn{2}{|c|}{ S. sodomeum (spontanée) } & 1 lot & 225 & (2) & 1 lot & 65 & (2) \\
\hline \multicolumn{2}{|c|}{ S. macrocarpon } & 1 lot & 7,5 & (3) & 1 lot & 30 & (4) \\
\hline \multicolumn{2}{|c|}{ S. aethiopicum (gr. Kumba) } & 1 lot & traces & & 1 lot & 40 & (2) \\
\hline \multirow{2}{*}{\multicolumn{2}{|c|}{ S. aethiopicum (gr. aculeatum) }} & 1 lot & 12,5 & (4) & 1 lot & 50 & (7) \\
\hline & S. aethiopicum (gr. Gilo) & 1 lot & 3,0 & (2) & 1 lot & 30 & (5) \\
\hline
\end{tabular}

(a) Moyenne des 2 dosages par colorimétrie et CCM du Tableau I. (b) Maturité dépassée evou valeur maximale trouvée. (c) Les analyses de solanacées voisines de l'aubergine (Solanum melongena $\mathrm{L}$.) portent sur un seul lot.

Tableau III. Influence des graines sur la teneur des fruits en saponosides.

\begin{tabular}{|c|c|c|c|c|c|c|c|c|c|}
\hline Variétés & \multicolumn{3}{|c|}{ "Claresse” } & \multicolumn{3}{|c|}{ «Konzervnij»" } & \multicolumn{3}{|c|}{ «Trachietz» } \\
\hline Pollinisation & C & $\mathrm{FL}$ & V & $\mathrm{C}$ & $\mathrm{FL}$ & V & C & $\mathrm{FL}$ & V \\
\hline Présence de graines & Sans & Faible & Forte & Sans & Sans & Forte & Sans & Faible & Forte \\
\hline $\begin{array}{l}\text { Couleur des fruits } \\
\text { UDO } 550 \mathrm{~cm}^{2}\end{array}$ & 1,4 & 1,6 & 1,6 & 5,7 & 4,5 & 3,9 & 3,1 & 3,9 & 3,3 \\
\hline $\begin{array}{l}\text { Glycoalcaloïdes (GA) } \\
\text { Colorimétrie } \\
\text { CCM }\end{array}$ & $\begin{array}{l}18,7 \\
12,0\end{array}$ & $\begin{array}{r}10,6 \\
6,8\end{array}$ & $\begin{array}{l}26,5 \\
20\end{array}$ & $\begin{array}{r}12,9 \\
8,0\end{array}$ & $\begin{array}{l}12,9 \\
10,4\end{array}$ & $\begin{array}{l}16,6 \\
11,5\end{array}$ & $\begin{array}{r}12,9 \\
8,5\end{array}$ & $\begin{array}{l}7,3 \\
5,6\end{array}$ & $\begin{array}{l}9,6 \\
7,5\end{array}$ \\
\hline $\begin{array}{l}\text { Répartition des taches } \\
\text { (Evaluation GA / CCM) }\end{array}$ & $\begin{array}{l}20 \\
10\end{array}$ & $\begin{array}{r}12 \\
5\end{array}$ & $\begin{array}{l}35 \\
15\end{array}$ & $\begin{array}{l}8 \\
6\end{array}$ & $\begin{array}{l}9 \\
8\end{array}$ & $\begin{array}{r}10 \\
7\end{array}$ & $\begin{array}{l}8 \\
6\end{array}$ & $\begin{array}{l}6 \\
4\end{array}$ & $\begin{array}{l}7 \\
6\end{array}$ \\
\hline en $\mu g$ par tache & - & - & $\begin{array}{l}- \\
-\end{array}$ & $\begin{array}{l}5 \\
1\end{array}$ & $\begin{array}{l}7 \\
2\end{array}$ & $\begin{array}{l}9 \\
3\end{array}$ & $\begin{array}{l}6 \\
1\end{array}$ & $\begin{array}{c}4 \\
\text { traces }\end{array}$ & $\begin{array}{l}5 \\
1\end{array}$ \\
\hline $\begin{array}{l}\text { Saponosides non azotés } \\
\text { (SNA) type furostanol/ } \\
\text { CCM }\end{array}$ & 5,6 & 6,0 & 6,2 & 2,4 & 1,2 & 1,2 & 3,2 & 2,8 & 3,2 \\
\hline
\end{tabular}




\section{DISCUSSION}

\section{Différences variétales et conditions de récolte}

L'étude systématique des récoltes successives d'un même essai montre, à la fois, l'augmentation des teneurs en glycoalcaloödes (GA) en fin de culture (Fig. 1) et l'existence de fluctuations périodiques en dents de scie, pour les GA (Fig. 1) comme pour les SNA (Fig. 2). Ces fluctuations peuvent correspondre à une hétérogénéité de stade de maturité physiologique des fruits, d'une récolte à l'autre. Mais elles peuvent aussi être le fait de variations métaboliques dans le fruit, qui sont tributaires de la charge de la plante et de la fréquence des cueillettes. Ainsi, l'espacement des récoltes en fin de saison est peut-être à l'origine des teneurs en glycoalcaloïdes relativement élevées pour les variétés analysées (Fig. 1). Inversement, le resserrement des dates de récolte abaisse les teneurs en glycoalcaloïdes, pour la plupart des variétés (cas des récoltes 5,6 et 8 ). L'espacement ou la suppression d'une récolte (cas de la récolte 4 pour "Athéna") entraîne une remontée (Figs 1 et 3).

En fait, la saveur des aubergines est certainement conditionnée à la fois par des facteurs génétiques, mais aussi, par les conditions de récolte. Malgré l'homogénéité des conditions de culture et la bonne concordance des résultats obtenus par 2 voies analytiques différentes, le classement fait entre génotypes situés entre les 2 cas extrêmes, "Ronde de Valence" et "Dourga", reste aléatoire étant donné la forte variabilité de la teneur en saponosides d'une série de mesures à l'autre (Tableau I). Des interprétations

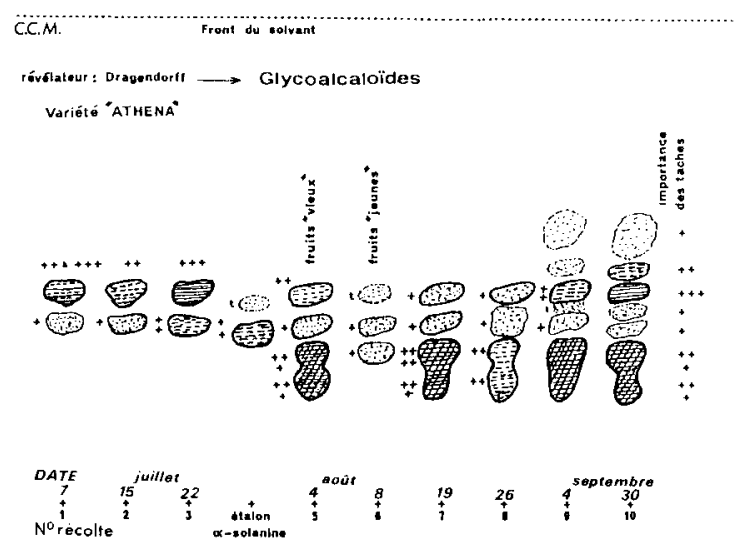

Fig. 3. Séparation par chromatographie sur couche mince (CCM) des hétérosides de la solasodine de l'aubergine : extraits des récoltes successives de la variété "Athéna", sur support de silice (G 1500 de Schleicher et Schull) avec l'éluant BAE (4-1-5 V) et le réactif de Dragendorff. hâtives devront être considérées avec beaucoup de circonspection. Ainsi, les forts maximums de certains lots, récoltés trop tardivement, augmentent considérablement les moyennes de ces variétés et faussent les confrontations. La saveur, utilisée comme critère de sélection, doit être l'objet d'impératifs d'échantillonnage particulièrement rigoureux.

Les espèces de Solanum phylogénétiquement proches de l'aubergine, ne nécessitent pas autant de précautions dans le choix des lots de fruits, car elles possèdent de fortes teneurs en saponosides stéroïdiques (Tableau II). C'est, en particulier, le cas de $S$. sodomeum, très riche à la fois en GA et en SNA, et dont la consommation est considérée traditionnellement comme dangereuse.

Mais d'autres espèces à fruits amers, comme $S$. macrocarpon et $S$. aethiopicum, gr. gilo ou gr. kumba, sont impunément consommées. Nos analyses montrent que ces espèces contiennent relativement peu de glycoalcaloïdes (teneurs voisines de celles qu'on trouve chez l'aubergine) et relativement plus de saponosides non azotés type furostanol. Dans le cas du "Djackattou" (S. aethiopicum gr. kumba), des préparations culinaires en Afrique de l'Ouest, ont limité, de façon empirique, la proportion de ce légume-condiment jusqu'au seuil d'acceptabilité gustative (De Bon, 1984).

\section{Influence de la quantité de graines conte- nues dans le fruit}

L'expérience dont les résultats sont consignés dans le Tableau III montre que l'aptitude à la parthénocarpie n'apporte pas une amélioration de la qualité gustative des fruits. Les fruits sans graines ont des teneurs en saponosides voisines de celles des fruits qui contiennent des graines. Ces résultats sont apparemment contradictoires avec des observations antérieures (Aubert et al., 1989) relatives à la plus faible teneur en GA et en SNA de la pulpe du fruit dépourvue de graines (zone située à proximité du pédoncule), par rapport à la pulpe contenant les graines (mésocarpe placentaire). Ces 2 séries de résultats ne sont cependant pas tout à fait comparables, car ils ont été obtenus sur des groupes variétaux différents, issus, de plus, de cultures différentes (abri et plein-champ).

Dans l'état actuel de l'avancement de nos travaux, un seul fait est net : la pulpe du fruit contient toujours des saponosides stéroïdiques, qu'elle contienne des graines ou pas. La détermination de l'influence exacte de la quantité de graines du fruit sur la teneur en saponosides 
nécessite d'autres expérimentations plus systématiques.

\section{Autres sources de variation et perspectives expérimentales}

L'influence des conditions agroclimatiques sur la synthèse des saponosides stéroïdiques semble considérable. L'accumulation de ces substances chez les solanacées soumises à des stress de diverses origines est étudiée par des physiologistes (Heftmann, 1983; Man et al., 1980).

Khristov (1975) sur l'aubergine, et Yaniv et al., (1984) sur d'autres solanacées, ont signalé la coïncidence entre longues périodes de sécheresse et accumulation de glycoalcaloïdes : la régularité des apports d'eau par irrigation semble favoriser l'établissement d'une faible teneur responsable d'une saveur douce.

Cependant, les différences variétales d'adaptation à la sécheresse climatique chez l'aubergine (Daunay, 1986; Pochard 1973, Pochard \& Serieys, 1974; Pochard \& Cornillon, 1975) ne peuvent être associées avec les différences de saveur. En effet, on trouve (Tableau III), à la fois des variétés sapides (type "Violette de Barbentane") et douces (type "Dourga"), bien adaptées à la sécheresse, et d'autres qui résistent mal à de telles conditions (comme "Liu Ye Qié" et "Shinkuro"), mais qui sont de saveur relativement douce.

Dans notre essai de variétés parthénocarpiques (Tableau III), nous avons relevé de fortes teneurs en saponosides, plus particulièrement en glycoalcaloïdes (jusqu'à 26,5 mg pour $100 \mathrm{~g}$ de matière fraîche). Elles sont probablement dues à la fois au stade de maturité des fruits, récoltés trop tard, et à un ensemble de conditions climatiques défavorables, notamment de fortes amplitudes thermiques jour-nuit et une certaine surchauffe diurne de l'air par manque d'aération de la serre.

Divers autres agents de stress peuvent modifier les teneurs en glycoalcaloïdes (Rowan et al., 1983). On note une augmentation des teneurs chez diverses solanacées à la suite de blessures, d'altérations pathologiques ou d'une perte du feuillage (Moutot, 1976).

Les hormones de croissance peuvent avoir des effets variables. Heble et al. (1971) signalent une augmentation des teneurs. Au contraire, Jadhav et al. (1973) enregistrent une diminution avec l'utilisation de détergents, d'inhibiteurs ou de réducteurs de croissance. Les traitements phytosanitaires, en particulier les nématicides, favoriseraient la biosynthèse des alcaloïdes (Bajaj \& Mahajan, 1980).
Tous ces facteurs de variation ouvrent de nouvelles voies et perspectives expérimentales pour préciser plus exhaustivement les différences de qualité gustative et sanitaire des aubergines.

\section{CONCLUSION}

L'amélioration de la qualité gustative de l'aubergine dépend, en premier lieu, de la maîtrise des conditions de culture et du stade physiologique auquel les fruits sont récoltés. L'influence du génotype sur les teneurs en saponosides des fruits est bien réelle, mais semble de moindre importance pour les diverses variétés analysées dans cette étude. D'autres espèces de Solanum, voisines de l'aubergine, se distinguent par leurs teneurs différentes en glycoalcaloïdes ou en saponosides non azotés du type furostanol; elles se caractérisent par leur saveur amère accentuée.

L'influence de la quantité de graines n'a pu être établie clairement, compte tenu de la perturbation de ces expériences par des conditions agroclimatiques particulièrement défavorables. Une meilleure connaissance des rôles respectifs des différents paramètres qui peuvent intervenir nécessiterait des expérimentations en milieux contrôlés et contrastés, par exemple pour les ressources en eau. Mais d'autres facteurs de variation des teneurs en saponosides devraient être considérés, notamment ceux susceptibles d'activer ou d'inhiber le métabolisme de ces substances.

Le développement d'un travail de sélection visant à créer des génotypes à faibles teneurs en glycoalcaloïdes et en saponosides non azotés nécessite, au préalable, une amélioration des techniques de séparation et de dosages individuels de ces composés.

Pratiquement, dans l'état actuel des connaissances et des méthodes d'analyse, la qualité gustative des aubergines pourrait déjà être nettement améliorée si les récoltes étaient fréquentes, dans des cultures régulièrement irriguées, et si les fruits à surmaturité commerciale étaient systématiquement éliminés.

\section{RÉFÉRENCES}

Aubert S. (1971) L'aubergine (Solanum melongena L.). Compositions et facteurs de qualité. Ann. Technol. Agric. 20, 241-264

Aubert S., Daunay M.C. \& Pochard E. (1989) Saponosides stéroïdiques de l'aubergine (Solanum melongena L.). I. Intérêt alimentaire, méthodologie d'analyse, localisation dans le fruit. Agronomie 9, 641-651 
Bajaj K.L. \& Mahajan R. (1980) Effects of nematicides on the chemical composition of the fruits of egg plant (Solanum melongena L.). Qual. Plant. Plant Foods Hum. Nutr. 30, 68-72

Daunay M.C. (1986) Adaptation de l'aubergine au climat méditerranéen : recherche de caractères morphologiques et physiologiques impliqués. Thèse de docteur ès sciences (développement et amélioration des végétaux). Faculté des sciences et techniques de Saint Jérome, Aix-Marseille III. (148 p., figures et tableaux 100 p.)

De Bon A. (1984) Description et culture d'une solanacée légumière de l'Ouest africain : le djackattou (Solanum aethiopicum L.). Agron. Trop. 39, 67-75

Defago G. (1977) Rôle des saponines dans la résistance des plantes aux maladies fongiques. Bull. Soc. Bot. Suisse 87, 79-132

Heble M.R., Narayanaswami S. \& Chadha M.S. (1971) Hormonal control of steroid synthesis in Solanum xanthocarpum tissues cultures. Phytochemistry 10, 2393-2394

Heftmann E. (1983) Biogenesis of steroid in solanaceae. Phytochemistry 22, 1843-1860

Hepper F.N. (1979) On typifying Linnaean names of Solanaceae. In: The Biology and Taxonomy of the Solanaceae. (J.G. Halukes, R.N. Lester \& A.D. Skelding, eds) Linnean Society Symposium series, (7) col. Academic Press, pp. 131-133

Jadhav S.J., Salunkhe D.K., Wyse R. \& Dalvi R.R. (1973) Solanum alkaloids biosynthesis and inhibition by chemicals. J. Food Sci. 38, 453-455

Joubert G. \& Compagnon P. (1974) Les Variétés de Poivron et d'Aubergine Cultivées en France. CTIFL Documents $n^{\circ} 42$

Juvik J.A., Stevens M.A. \& Rick C.M. (1982) Survey of the genus $L y c o p e r s i c o n$ for variability in alpha-tomatine content. Hortscience, 17, 764-766
Khristov S. (1975) Egg Plants - Kach. Prom. Zelenchokow Sortove. (Mikkov, ed.) Khristo G. Danov, Plovdiv, Bulgarie, pp. 125-133; CR Acad Sci Agric Bulg 15, 74933

Lester R.N. \& Niakan L. (1986) Origin and domestication of the Scarlet Egg plant, Solanum aethiopicum L. from S. anguivi Lam. In: Solanaceae : Biology and Systematics. (W.G. D'Arcy ed.), Columbia University Press, New York, pp. 433-456

Man J.D., Edge E.A., Lancaster J.E. \& Blyth K. (1980) Growth and solasodine production by Solanum aviculare and Solanum lacianatum under moisture stress at different temperatures. NZJ Agric. Res. 23, 361-366

Moutot F. (1976) Les glycoalcaloïdes des solanacées. Mémoire bibliographique (27 p., 554 réf.), INRA Versailles.

Pochard E. (1973) Sélection de l'aubergine pour la culture en serre. Mise en évidence de différences variétales liées à l'état hydrique des plantes. Acta Hortic. (The Hague) 42, 123-142

Pochard E. \& Serieys H. (1974) Mise en évidence de différences variétales de transpiration chez l'aubergine et d'autres solanées (Solanum melongena L., Capsicum annuum L., Lycopersicum esculentum Mill.)Conséquences pour l'adaptation à différents climats. Ann. Amélior. Plant. 243-268

Pochard E. \& Cornillon P. (1975) Influence de l'état hydrique des plantes sur la croissance et la fructification du piment et de l'aubergine en serre. Acta Hortic. 51, 175-184

Rowan D.D., MacDonald P.E. \& Skipp R.A. (1983) Antifungal stress metabolites from Solanum aviculare. Phytochemistry 22, 2102-2104

Yaniv Z., Palevitch D. \& Weissenberg M. (1984) The effect of water stress on growth and solasodine content in Solanum khasianum. Planta Medica 50, 6065 